\title{
Knowledge, attitude and perception regarding antimicrobial resistance and usage among ruminant farmers in Selangor, Malaysia
}

\begin{abstract}
The increasing public health problem of antimicrobial resistance (AMR) has been linked to the extensive antimicrobial use (AMU) in food animals. We conducted a survey among ruminant farmers in Selangor, Malaysia to assess their level of awareness on AMR, attitudes towards AMU, and determinants that influence their practices. The survey was developed in English and Malay, validated, and administered to ruminant farmers in Selangor. A total of 84 farmers (response rate of 55\%) completed the structured questionnaire. They appeared to be little aware of AMR and the impact on animals and public health. Indications of inappropriate AMU include their misconception on conditions requiring antibiotic therapy and easy accessibility to antibiotics. More than $70 \%(60 / 84)$ of the respondents believed that all sick animals need to be given antimicrobials. Half of the farmers especially those involved in the production of small and large ruminants; namely mixed ruminant farmers (MRF) $(63 \%, 31 / 49)$ indicated that antimicrobials do not have any side effects in animals. Sixty-four percent (54/84) of the farmers have stored antimicrobials in their farms of which the practice was more common $(\mathrm{P}=0.02)$ among the MRF compared to the single ruminant farmers (SRF). Although most of the farmers felt good farm biosecurity will help reduce AMU, they were indifferent regarding using antimicrobials only when prescribed by a veterinarian and non-storage of antimicrobials for later uses. Farmers with larger herd size (>100 animals/herd) and few years of farming experience agreed more to the suggestions about their role and that of veterinarians respectively in reducing the drivers of AMR. These areas might need to be considered by advisors to inform ruminant farmers on AMR and to encourage them for prudent AMU in food-producing animals.
\end{abstract}

Keyword: Antimicrobial resistance; Antimicrobials; Antibiotics; Farmers; Ruminants 